\title{
Mortalidade infantil no Brasil: Belíndia ou Bulgária?
}

\author{
Infant mortality rate in Brazil: \\ Belgium-India or Bulgaria?
}

Célia Landmann Szwarcwald 1

Maria do Carmo Leal 2

Euclides Ayres de Castil ho 1

Carla L. Tavares de Andrade 1

\footnotetext{
1 Departamento de Informações para a Saúde, Centro de Informações Científico-Tecnológicas, Fundação Oswaldo Cruz. Av. Brasil 4.365, Rio de Janeiro, RJ 21045-900, Brasil. 2 Departamento de Epidemiologia, Escola Nacional de Saúde Pública, Fundação Oswaldo Cruz. Av. Leopoldo Bulhões 1.480, Rio de Janeiro, RJ 21041-210, Brasil.
}

Abstract In this paper, weanalyze the infant mortality in Brazil in the 1980 decade. Since the completeness of death registration is very low in some regions of the country, the analysis is based on five health indicators constructed from the proportional distribution by causes of death and by age-group. Using a principal component analysis, we develop an index that expresses quantitatively the diversified conditions of mortality. Through this index, classifying the Brazilian states in three groups, it is shown that the poorest one continues to exhibit a pattern similar to India and other low income populations. Additionally, analyzing the neonatal mortality in the weal thiest group, it is found a small reduction in the first week mortality, indicating that even the favored group still remains very far from the devel oped world. In general, when compared to other countries, Brazil has shown a poor performance. It becomes evident the need of reestablishing strategies to improvement on both components, the neonatal and the post-neonatal mortality.

Key words Infant Mortality; Neonatal Mortality; Causes of Death; Heal th Indicators; Epidemiology

Resumo Nestetrabal ho, descreve-se a evolução da mortalidade infantil no Brasil na década de 80. Diante da el evada proporção de óbitos não registrados no País, a caracterização do risco de morrer entre as crianças menores de um ano de idade é realizada por um conjunto de indicadores de saúde el aborados com base na distri bui ção por causa de morte e por componente etário. Por meio de uma análise estatística por componentes principais, desenvolve-se um índice sintetizador que permite expressar quanti tati vamente as diferentes situações de saúde nas Unidades Federadas. Através desta escala de valores, dividindo-se o território nacional em três grandes grupos, demonstra-se que a parte mais pobre persiste com padrão similar ao da Índia. Adicionalmente, a análise da mortali dade neonatal nos estados mais desen vol vidos mostra redução pouco expressi va nos coefici entes na primeira semana de vida, demonstrando-se que o padrão observado, mesmo nos estados em situação privilegi ada em relação aos demais, sequer se aproxima do que ocorre no mundo desenvolvido. De manei ra geral, o confronto com a experiência internaci onal leva a constatar que o declínio da mortali dade infantil na década de 80 foi pobre, ficando evi dente que o rumo a ser seguido envolve intervenções específicas sobre ambos os componentes, o neonatal e o tardio.

Palavras-chave Mortalidade Infantil; Mortalidade Neonatal; Causas de Morte; Indicadores de Saúde; Epidemiologia 


\section{Introdução}

O nível de qualidade de vida de uma sociedade é difícil de ser mensurado em sua plenitude. Contudo, pode ser avaliado por meio de indicadores em determinadas áreas consideradas como componentes essenciais para o delineamento do quadro de bem-estar social de uma população (The World Bank, 1995).

No âmbito da saúde pública, a evolução das chamadas estatísticas de saúde tem sido, constantemente, fonte de análise para caracterizar as condições de vida de uma população (Hanoluwka, 1987). Dentre os indicadores, o mais tradicional é o coeficiente de mortalidade infantil. Por ser, reconhecidamente, aquele que expressa não só o nível de saúde de uma sociedade, mas também o seu padrão sócio-econômico, tem sido universal mente utilizado como um índice sintetizador de desenvolvimento (Laurenti et al., 1985).

Entretanto, a precariedade das estatísticas de Registro Civil, tanto de nascimentos, como de óbitos, tem imposto grandes limitações para o cálculo deste indicador no Brasil. Seja utilizando como fontes de dados os censos demográficos ou pesquisas especiais, seja corrigindo as próprias informações de registro, é sempre preciso recorrer a métodos indiretos de estimação. Dependendo da técnica utilizada, os resultados são, muitas vezes, díspares e dificultam o conhecimento do nível real da mortalidade infantil no País.

Assim é que, no início da década de 80 , a Fundação Instituto Brasileiro de Geografia e Estatística (FIBGE) apontava para um decréscimo no risco de morrer entre os menores de um ano, de $87 / 1.000$ para $68 / 1.000$ nascidos vivos (NV), entre 1980 e 1984 (Simões \& Oliveira, 1986), baseando-se em informações do censo demográfico e da Pesquisa Nacional de Amostra por Domicílios (PNAD) para os referidos anos. No mesmo ano de 1984, o Ministério da Saúde, valendose da análise de dados de registro, indicava um aumento na mortalidade infantil, tido como um efeito da crise econômica atravessada pelo Brasil neste período (Becker \& Letchig, 1986).

Em recente Relatório sobre o Desenvolvimento Humano no Brasil (Ipea, 1996), publicado para o Programa das Nações Unidas para o Desenvolvimento (PNUD), proclamou-se, para a década de 90 , um coeficiente de mortalidade infantil (CMI) da ordem de 40/1.000 NV, acusando um decréscimo de mais de $100 \%$ neste indicador na última década. Adicionalmente, por meio da construção de um índice de desenvolvimento humano, baseado em três indicadores relacionados, respectivamente, à educa- ção, à longevidade e ao acesso a recursos, as Unidades da Federação (UF) foram classificadas em três grandes grupos. À clássica Belíndia de Bacha \& Klein (1986), acrescentou-se um grupo intermediário, incluindo os Estados de Rondônia, Amazonas, Roraima e Amapá, da Região Norte; Goiás e Mato Grosso, da Região Centro-Oeste, e Minas Gerais, da Região Sudeste, que foi equiparado à Bulgária em termos de desenvolvimento humano.

Diante da reconhecida sensibilidade da mortalidade infantil para expressar o padrão de vida de uma população, e tendo-se em conta que o CMI de 40/1.000 NV não expressa, de fato, a realidade brasileira, este trabal ho tem como propósito analisar os óbitos entre as crianças menores de um ano notificados no período de 1979 a 1993. Por meio da construção de indicadores de saúde, elaborados com base na distribuição por causa de morte e na distribuição por componente etário entre os óbitos de menores de um ano de idade, desenvolve-se um índice sintetizador que permite expressar quantitativamente as diferentes situações da mortalidade infantil no Brasil.

Através desta escal a de valores, dividindose o País em três grandes grupos de Unidades Federadas, demonstra-se que o grupo mais pobre persiste com padrão semelhante ao Índia. Porém, nem o grupo intermediário se identifica à Bulgária, nem o grupo de estados mais desenvolvidos se parece com a Bélgica. Pelo contrário, a análise da tendência dos óbitos neonatais nos Estados do Rio de Janeiro, São Paulo e Rio Grande do Sul leva a crer que o padrão observado sequer se aproxima do que ocorre no mundo desenvolvido.

\section{O sub-registro de óbitos}

A divulgação das informações de registro dos eventos vitais no Brasil é de responsabilidade da Fundação Instituto Brasilei ro de Geografia e Estatística (FIBGE). A partir de 1974, as estatísticas de nascimento e óbito vêm sendo publicadas anualmente sob o título Estatísticas do Registro Civil, sendo 1993 o último ano com informações disponíveis (FIBGE, 1981 a 1995).

O Sistema de Informações sobre Mortalidade do Ministério da Saúde (SIM/MS) constituise em uma fonte alternativa de dados de óbitos no País (MS, 1995). Criado em 1976, a partir da implantação do modelo padronizado da declaração de óbito (DO) em todo o território nacional, é justificado não só para o atendimento de exigências legais. Com o objetivo principal de fornecer subsídios para análises de mortalida- 
de, o SIM / MS apresenta estatísticas calculadas a partir da classificação da causa básica da morte (MS, 1982).

O fluxo das estatísticas de mortalidade tem início com o registro do óbito em cartório, mediante a apresentação da DO preenchida em duas vias. As informações divulgadas pela FIBGE são provenientes da transcrição das informações das DOs em mapas mensais pelos Cartórios de Registro Civil que são enviados trimestral mente às superintendências estaduais do IBGE e destas para o nível central.

Já o SIM/MS tem como base do sistema as informações individualizadas das próprias DOs. Após a coleta dos dados, as DOs são classificadas por município de residência do falecido, passando-se, a seguir, à seleção da causa básica do óbito, segundo os critérios internacionais recomendados pela Organização Mundial de Saúde (CCDP, 1978). Em nível nacional, as informações disponíveis são referentes aos anos de 1977 a 1991, apesar de alguns estados possuírem dados mais recentes.

Apesar da disponibilidade de dois sistemas de informações sobre óbitos em nível nacional, um dos grandes problemas que ainda permeiam as análises de mortalidade no Brasil é o sub-registro de óbitos, que tem magnitude expressiva sobretudo nas regiões menos desenvolvidas.

Ao publicar, pela primeira vez, as Estatísticas de Mortalidade relativas ao ano de 1979, o Ministério da Saúde (MS, 1982) referia que o Sistema de Informações sobre Mortalidade (SIM/MS), de abrangência nacional, incluía apenas cerca de $70 \%$ do total de óbitos ocorridos naquele ano. Os dados mais recentes não mostram qualquer indicação de melhora na cobertura do registro dos óbitos. Pelo contrário, alguns estados têm apresentado, inclusive, deterioração das informações de mortalidade.

A subenumeração de óbitos diz respeito, principalmente, à ocorrência de sepultamentos sem a exigência da certidão, nos denominados cemitérios clandestinos. Pesquisas realizadas no Sul e no Nordeste do País comprovaram a existência de vários cemitérios, não oficiais, com enterramentos sem a documentação necessária (Barros et al., 1985; Mello Jorge, 1983; Mello Jorge, 1987).

O sub-registro de óbitos no Brasil é diferenciado por região - sendo superior no Norte e no Nordeste - e por idade, com predomínio entre os menores de um ano. Tem grandeza tão relevante na grande maioria das Unidades da Federação, que torna inviável a estimativa dos indicadores de mortalidade através da sua fórmula definidora.
A constatação deste fato pode ser verificada por meio da análise dos coeficientes gerais de mortalidade calculados segundo municípios, para 1991, pelas estatísticas do SI M/ MS e do último censo demográfico. Na Tabela 1, são apresentadas as distribuições regionais dos municípios classificados segundo o valor do coeficiente geral de mortalidade. No Norte, Nordeste e Centro-Oeste mais de $50 \%$ dos municípios têm taxas menores do que 4/ 1.000 habitantes, expressão provável de um grau muito alto de subnotificação. Quanto aos locais com coeficientes que variam de 4/ 1.000 a 6/ 1.000 habitantes, é difícil precisar se há expressivo sub-registro ou se a taxa de mortalidade geral é efetivamente pequena. Porém, a concentração de municípios nesta categoria na Região Sul, que tem, reconhecidamente, elevado percentual de idosos, fornece evidências de sub-notificação. A maior proporção de municípios com coeficientes maiores do que 6/ 1.000 é apresentada pela Região Sudeste. Em relação à Região Nordeste, convém notar que, embora $21 \%$ dos municípios tenham coeficientes superiores a $6 / 1.000$, certamente, aqueles que melhor notificam a mortalidade, estes podem ainda ter coberturas não completas, já que em regiões pobres as taxas de mortalidade geral esperadas são bastante elevadas.

Ainda que coeficientes gerais de mortalidade sejam dependentes da estrutura etária da população, pois regiões com maior proporção de idosos apresentam, em geral, maiores coeficientes, evidencia-se que, mesmo tomando-se como limite $4 / 1.000$ habitantes, é muito grande o percentual (35\%) de municípios brasileiros com coeficiente de mortalidade notificada inferior a este valor.

Sendo a magnitude do sub-registro diferenciada por região, é importante estabelecer que análises para o território nacional não podem ser feitas diretamente, mas corrigindo-se, pri-

Tabela 1

Distribuição Percentual (\%) dos municípios segundo o valor do coeficiente geral de mortalidade por Grande Região. Brasil, 1991.

\begin{tabular}{lccc}
\hline Região & $\begin{array}{c}\text { Coeficiente Geral de } \\
0,0-4,0\end{array}$ & $4,1-6,0$ & 6,1 e mais \\
& 84,93 & 13,24 & 1,84 \\
Norte & 53,34 & 25,21 & 21,45 \\
Nordeste & 12,78 & 39,82 & 47,40 \\
Sudeste & 14,20 & 53,29 & 32,51 \\
Sul & 56,37 & 36,59 & 7,05 \\
Centro-O este & 34,52 & 35,77 & 29,71 \\
Brasil & & & \\
\hline
\end{tabular}


meiramente, as informações regionais pelos devidos fatores de correção do sub-registro. Como a subenumeração é bem maior no Norte e no Nordeste, os dados nacionais sem correção expressam preponderantemente a situação de mortalidade das regiões mais desenvolvidas.

O mesmo raciocínio é válido para os dados do litoral do Nordeste em relação ao sertão nordestino, das capitais em relação ao interior dos estados, dos centros urbanos em relação às áreas rurais. As informações de registro vão sempre corresponder às áreas que melhor notificam a mortalidade.

Chama-se a atenção para este fato. Ainda que se corrijam as informações de registro por fatores diferenciados por região, estar-se-ão corrigindo apenas aquelas referentes às áreas que, de al guma forma, notificam a mortalidade eque são, possivelmente, as que têm as melhores situações de saúde.

A mortalidade infantil no início da década de 90

Diante do sub-registro expressivo entre os óbitos de menores de um ano no Brasil, métodos indiretos de estimação têm sido utilizados para fornecer valores aproximados da mortalidade infantil em anos recentes.

Valendo-se de um método baseado na curva de mortalidade proporcional por idade obtida pelas estatísticas de registro civil, Szwarcwald \& Castilho (1995) estimaram a mortalidade infantil para o Brasil, na década de 80. As projeções destas estimativas para 1990 e 1991 são respectivamente de 58/ 1.000 e 56/ 1.000 NV.

Embora com metodologia e fonte de informações diferentes, o Banco Mundial fornece em seus relatórios anuais estimativas bem semelhantes (Banco Mundial, 1993; 1994; 1995). As estimativas estabelecidas para os anos de 1990, 1991 e 1992 são respectivamente de 57/ $1.000,58 / 1.000$ e $57 / 1.000$ NV.

Supõe-se, assim, que este patamar (entre $55 / 1.000$ e $60 / 1.000 \mathrm{NV}$ ) seja aquele que meIhor represente o nível da mortalidade infantil no Brasil no início da década de 90 . Contudo, mesmo sem entrar no mérito ou na sofisticação dos métodos demográficos, apenas um raciocínio intuitivo leva, da mesma forma, a concluir que o CMI nacional é bem superior ao valor de 40/ 1.000 NV, divulgado pel o I pea (1996) ao caracterizar o perfil de saúde da população brasileira.

Primeiramente, como o Estado de São Paulo apresentava um CMI de 27/ 1.000 NV para
1991 (Seade, 1993), é razoável supor que a parte mais desenvolvida do País tivesse, pelo menos, uma taxa de 30/1.000 NV no início dos anos 90 . Sendo assim, para o valor médio de 40/ 1.000 NV para o Brasil, seria preciso que a Região Nordeste tivesse um coeficiente em torno de 50 , o que, reconhecidamente, está muito aquém da realidade nordestina.

\section{Indicadores marcadores da situação de saúde}

Devido ao grande problema encontrado no cálculo direto da mortalidade infantil, para caracterizar as diferentes situações de saúde no País, foi necessária a elaboração de indicadores que pudessem ser construídos por meio dos dados disponíveis, ou seja, que, sob determinadas suposições, fossem invariantes ao sub-registro de óbitos.

De maneira geral, os óbitos infantis podem ser classificados em dois grandes grupos, de acordo com a idade da criança. O componente neonatal, quando a morte ocorre dentro das quatro primeiras semanas de vida, que está associado principalmente às causas perinatais e às anomalias congênitas, e o componente pósneonatal ou tardio, que relaciona-se predominantemente às doenças infecciosas e à desnutrição (Pollard et al., 1974).

$\mathrm{Na}$ Tabela 2, apresenta-se a distribuição proporcional por idade dos óbitos de menores de um ano, em alguns países selecionados, desde os anos 60 até a década atual. Para todos os países sob análise, o comportamento é o mesmo. Nos primeiros anos estudados, constata-se uma relação direta entre a proporção de óbitos no período tardio e o valor do CMI: quanto maior o percentual de óbitos tardios, maior a taxa de mortalidade infantil. Em um período posterior, o declínio da mortalidade pós-neonatal, em velocidade maior que a precoce, provoca um aumento na proporção de mortes neonatais. Porém, anos mais tarde, os esforços dedicados à redução da mortalidade nas primeiras horas têm reflexos nos seus componentes, que passam por um processo de inversão, apresentando novamente aumento na proporção de óbitos tardios (Lee et al., 1980).

Em comparação com os dados brasileiros, apenas o Distrito Federal, São Paulo e Rio de Janeiro têm, na década de 90 , estatísticas compatíveis à Bélgica dos anos 60 , com proporção de óbitos tardios em patamar equivalente, menor do que $40 \%$ (Tabela 3). Chamam a atenção também os altíssimos percentuais encontrados na Região Nordeste. 


\section{Tabela 2}

Coeficiente de mortalidade infantil (CMI), componente neonatal precoce, componente pós-neonatal e proporção de mortes por diarréia para países e anos selecionados.

\begin{tabular}{|c|c|c|c|c|c|}
\hline País & Ano & $\begin{array}{l}\text { CMI } \\
(/ 1,000)\end{array}$ & $\begin{array}{l}\text { Proporção (\%) } \\
0-6 \text { dias }\end{array}$ & $\begin{array}{l}\text { Proporção (\%) } \\
\text { pós-neonatal }\end{array}$ & $\begin{array}{l}\text { Proporção (\%) } \\
\text { diarréia }\end{array}$ \\
\hline \multirow[t]{5}{*}{ J apão } & 1960 & 30,7 & 34,6 & 44,5 & 7,7 \\
\hline & 1970 & 13,1 & 50,4 & 34,1 & 3,6 \\
\hline & 1980 & 7,5 & 52,0 & 34,2 & 0,9 \\
\hline & 1986 & 5,2 & 44,8 & 40,8 & 0,3 \\
\hline & 1992 & 4,5 & 38,9 & 47,0 & 0,2 \\
\hline \multirow[t]{5}{*}{ Bélgica } & 1960 & 31,2 & 54,6 & 34,6 & 3,8 \\
\hline & 1970 & 21,1 & 57,6 & 32,6 & 1,1 \\
\hline & 1975 & 16,2 & 62,0 & 27,3 & 0,8 \\
\hline & 1979 & 12,3 & 56,0 & 33,4 & 0,9 \\
\hline & 1989 & 8,5 & 43,1 & 45,4 & 0,2 \\
\hline \multirow{5}{*}{ Estados Unidos } & 1960 & 26,0 & 64,1 & 28,1 & 2,8 \\
\hline & 1969 & 21,0 & 67,5 & 25,3 & 1,2 \\
\hline & 1974 & 16,7 & 63,9 & 26,6 & 1,3 \\
\hline & 1984 & 10,8 & 54,5 & 35,1 & 0,4 \\
\hline & 1991 & 8,9 & 51,5 & 37,5 & 0,4 \\
\hline \multirow[t]{5}{*}{ Portugal } & 1960 & 77,5 & 19,4 & 63,9 & 26,5 \\
\hline & 1971 & 49,8 & 31,7 & 54,7 & 20,9 \\
\hline & 1979 & 26,0 & 47,7 & 39,5 & 9,6 \\
\hline & 1985 & 17,8 & 57,4 & 31,8 & 3,8 \\
\hline & 1993 & 8,7 & 47,2 & 36,4 & 1,1 \\
\hline \multirow[t]{5}{*}{ Polônia } & 1960 & 56,8 & 31,1 & 54,9 & 8,7 \\
\hline & 1970 & 33,2 & 42,9 & 41,0 & 2,2 \\
\hline & 1980 & 21,3 & 49,1 & 37,4 & 2,7 \\
\hline & 1985 & 22,1 & 56,4 & 29,5 & 1,1 \\
\hline & 1994 & 13,4 & 53,5 & 28,3 & 0,3 \\
\hline \multirow[t]{5}{*}{ Bulgária } & 1961 & 37,8 & 27,3 & 53,9 & 2,6 \\
\hline & 1970 & 27,3 & 33,2 & 51,7 & 5,1 \\
\hline & 1980 & 20,2 & 36,9 & 48,8 & 2,4 \\
\hline & 1985 & 15,2 & 37,8 & 45,7 & 1,6 \\
\hline & 1993 & 15,5 & 39,9 & 43,8 & 1,8 \\
\hline \multirow[t]{3}{*}{ Chile } & 1974 & 63,3 & 27,1 & 59,3 & 14,0 \\
\hline & 1980 & 36,6 & 38,2 & 50,1 & 7,5 \\
\hline & 1989 & 17,1 & 42,0 & 46,6 & 2,2 \\
\hline
\end{tabular}

Fonte: World Health Organization (1963; 1973; 1976; 1982; 1987; 1992; 1995).

Além dos componentes etários, a mortalidade proporcional por causas de óbito entre os menores de um ano constitui-se igualmente em um outro conjunto de indicadores com grande sensibilidade à situação de saúde e às condições de vida de uma determinada população. É o caso da proporção de óbitos classificados na rubrica 'sintomas, sinais e afecções mal-definidas', que representa a fração das mortes em que não houve definição da causa básica de óbito e expressa, geralmente, a carência de assistência médica.

No Brasil, é alarmante a grande proporção de mortes mal-definidas. Os estados situados no Norte e no Nordeste, em sua grande maioria, apresentam mais de $20 \%$ das mortes entre os menores de um ano sem definição da causa básica (Tabela 3). 
Tabela 3

Indicadores de saúde segundo unidade da federação, 1991.

\begin{tabular}{|c|c|c|c|c|c|}
\hline UF & $\begin{array}{l}\text { Proporção (\%) } \\
\text { anomalias congênitas }\end{array}$ & $\begin{array}{l}\text { Proporção (\%) } \\
\text { diarréia }\end{array}$ & $\begin{array}{l}\text { Proporção (\%) } \\
\text { mal-definidas }\end{array}$ & $\begin{array}{l}\text { Cobertura } \\
\text { de registro (\%) }\end{array}$ & $\begin{array}{l}\text { Proporção (\%) } \\
\text { pós-neonatal }\end{array}$ \\
\hline RO & 5,7 & 18,7 & 11,8 & 53,0 & 52,6 \\
\hline$A C$ & 9,5 & 12,6 & 23,2 & 49,3 & 52,6 \\
\hline$A M$ & 6,0 & 20,4 & 39,7 & 46,1 & 54,3 \\
\hline $\mathrm{RR}$ & 7,1 & 7,8 & 9,0 & 43,6 & 44,5 \\
\hline PA & 6,4 & 22,4 & 22,9 & 45,6 & 50,8 \\
\hline$A P$ & 6,9 & 11,2 & 2,5 & 48,4 & 39,8 \\
\hline TO & 8,1 & 16,3 & 25,6 & 36,3 & 56,6 \\
\hline $\mathrm{MA}$ & 5,3 & 21,4 & 16,3 & 26,1 & 51,1 \\
\hline PI & 4,1 & 19,0 & 10,5 & 29,8 & 48,3 \\
\hline CE & 5,4 & 27,6 & 45,9 & 43,0 & 69,7 \\
\hline RN & 5,3 & 20,6 & 28,7 & 43,2 & 61,2 \\
\hline PB & 6,5 & 20,3 & 51,1 & 65,4 & 67,0 \\
\hline PE & 6,4 & 20,2 & 45,8 & 72,1 & 57,7 \\
\hline$A L$ & 2,4 & 25,8 & 54,4 & 57,5 & 74,9 \\
\hline SE & 4,6 & 10,9 & 35,8 & 50,0 & 51,5 \\
\hline BA & 5,4 & 18,7 & 25,2 & 47,6 & 64,9 \\
\hline MG & 8,9 & 8,3 & 8,2 & 88,8 & 39,9 \\
\hline ES & 11,3 & 8,3 & 16,4 & 84,0 & 44,7 \\
\hline RJ & 10,4 & 6,1 & 4,7 & 100,0 & 36,5 \\
\hline SP & 11,3 & 6,1 & 3,6 & 100,0 & 34,7 \\
\hline PR & 12,9 & 13,7 & 10,1 & 87,6 & 44,7 \\
\hline $\mathrm{SC}$ & 15,2 & 9,2 & 11,9 & 77,6 & 46,2 \\
\hline RS & 14,7 & 5,1 & 4,2 & 100,0 & 41,2 \\
\hline MS & 7,8 & 10,2 & 8,2 & 67,2 & 47,7 \\
\hline MT & 0,9 & 19,8 & 15,9 & 50,2 & 62,1 \\
\hline GO & 13,6 & 8,4 & 10,3 & 65,6 & 42,6 \\
\hline $\mathrm{DF}$ & 16,4 & 6,9 & 0,7 & 100,0 & 38,8 \\
\hline
\end{tabular}

Fonte: Ministério da Saúde (1995).

Importante marcador de situações de pobreza é o percentual de óbitos infantis atribuídos às doenças infecciosas intestinais. Associada à desnutrição, à falta de saneamento e à deficiência de cuidados primários, a mortalidade proporcional por diarréia, apresentada no território brasileiro segundo as Unidades Federadas (Tabela 3), aponta para as condições desumanas de vida em algumas áreas do País. É surpreendente observar que estados considerados com maior nível de desenvolvimento, como Paraná e Santa Catarina, ainda apresentam altas proporções de óbitos por diarréia. Convém notar, porém, que a análise comparativa no Brasil deve ser interpretada com as devidas reservas, já que, construído a partir dos óbitos que têm definição da causa básica de morte, este indicador pode estar sendo subestimado nas áreas com grandes percentuais de mortes mal-definidas.

A situação configurada na Tabela 3 revela toda a sua gravidade ao se confrontarem os dados nacionais aos de outros países (Tabela 2). Das populações selecionadas, o maior percentual de mortes por diarréias foi apresentado pelo Chile, ligeiramente superior a 2\%, em 1989.

Além da proporção de óbitos sem definição da causa básica e da mortalidade proporcional por diarréia, outros indicadores podem também contribuir para a caracterização das condições de saúde de uma dada conformação social. Os indicadores utilizados neste trabal ho como marcadores de condições de saúde entre os menores de um ano estão dispostos na primeira coluna da Tabela 4. Para a sua construção, supôs-se sub-registro homogêneo de mor- 
tes entre os menores de um ano, tanto por componente etário, como por causa de óbito. Em relação à mortalidade proporcional por causas, a distribuição foi obtida excluindo-se, do total de óbitos, aqueles com causa indefinida.

Para complementar o conjunto de variáveis, um indicador de cobertura do registro de óbitos foi também considerado. Diante da ausência de informações sobre a proporção de óbitos não registrados por UF, este indicador foi estimado de forma aproximada. Primeiramente, utilizando-se os fatores de correção de sub-registro estimados segundo as grandes regiões (Szwarcwald \& Castilho, 1995), calcularam-se os coeficientes gerais de mortalidade em cada uma das cinco regiões do País. Dividiu-se, então, a taxa observada de mortalidade de cada UF pela taxa corrigida da região de localização da UF para obter o indicador requerido. A exceção foi feita para os Estados do Rio de Janeiro, São Paulo, Rio Grande do Sul e para o Distrito Federal, cujos sistemas de registro foram considerados completos.

Todos os indicadores sob análise, apresentados por Unidade Federada, podem ser contemplados na Tabela 3.

\section{Um índice sintetizador}

A técnica estatística denominada de análise de componentes principais permite sintetizar o espaço multivariado a um número menor de dimensões, tornando mais clara a interpretação do problema através dos novos fatores (Green, 1978). O procedimento consiste em partir sucessivamente a variância total original. Ao primeiro componente cabe explicar a mai or parte sendo, por isto, denominado de índice sintetizador.

Este eixo unidimensional é composto por uma combinação linear dos indicadores originais. Quanto maior a correlação entre a variável original e o novo eixo, denominada de carga, tanto maior a sua representatividade no índice sintetizador.

Para sintetizar as variadas situações de mortalidade infantil encontradas nas Unidades da Federação, esta técnica foi utilizada, reduzindo o conjunto dos cinco indicadores em estudo a um único fator. O primeiro componente principal encontrado explica $75 \%$ da variância total e tem cargas grandes e positivas atribuídas à mortalidade proporcional por diarréias, à proporção de óbitos tardios e ao percentual de mortes mal-definidas (Tabela 4). Por outro lado, às anomalias congênitas e à cobertura de
Tabela 4

Cargas no primeiro componente principal.

\begin{tabular}{lc}
\hline Indicador & Carga \\
\hline 1. Proporção de óbitos por anomalias congênitas* & $-0,855$ \\
2. Proporção de óbitos por diarréia* & 0,936 \\
3. Proporção de óbitos sem definição da causa básica & 0,825 \\
4. Cobertura de registro de óbitos & $-0,766$ \\
5. Proporção de óbitos no período pós-neonatal & 0,924 \\
\hline
\end{tabular}

* Entre os óbitos com causa definida.

registro de óbitos correspondem cargas negativas de magnitude expressiva.

Os scores no índice sintetizador são os valores obtidos para cada unidade experimental no novo fator. Ou seja, são os resultados obtidos para cada UF na nova variável, composta linearmente pelas originais e capaz de sintetizar o conjunto das mesmas.

Sendo assim, os scores constituem-se em uma escala que permite quantificar, ainda que de forma relativa, as diferentes condições de saúde entre os menores de um ano encontradas no território brasileiro. Os scores do índice sintetizador obtidos segundo UF estão dispostos na Tabela 5, e os valores negativos representam as melhores situações,enquanto os positivos expressam as piores.

Classificando as UFs em quatro grandes grupos de acordo com esta escala, o mapa temático apresentado na Figura 1 descreve com tom mais claro as melhores situações de saúde, e com tonalidade um pouco mais escura os estados em posição intermediária e abaixo da média. Em sombreado escuro, estão aqueles em posição intermediária mas acima da média, e, em preto, os que têm péssimas e alarmantes condições de vida.

Na Tabela 6, estão dispostos os postos atribuídos aos cinco indicadores originais, numerados de um a 27 , ordenados, respectivamente, da melhor à pior situação. As UFs foram classificadas, da primeira à 27ạ posição, de acordo com o score obtido no índice sintetizador.

Vale ressaltar neste momento que, embora se tenha procurado minimizar os efeitos das deficiências dos dados, ao classificar as UFs através do índice sintetizador, não pode ser esquecido que este índice depende da qualidade das informações requeridas para a sua construção.

Alguns pontos, assim, devem ser levados em consideração. O mais importante refere-se ao sub-registro de óbitos. Conforme referido anteriormente, tendo-se estimado os indicadores por meio dos óbitos registrados, as estima- 
Figura 1

Situação da mortalidade infantil segundo unidade da federação. Brasil, 1991.

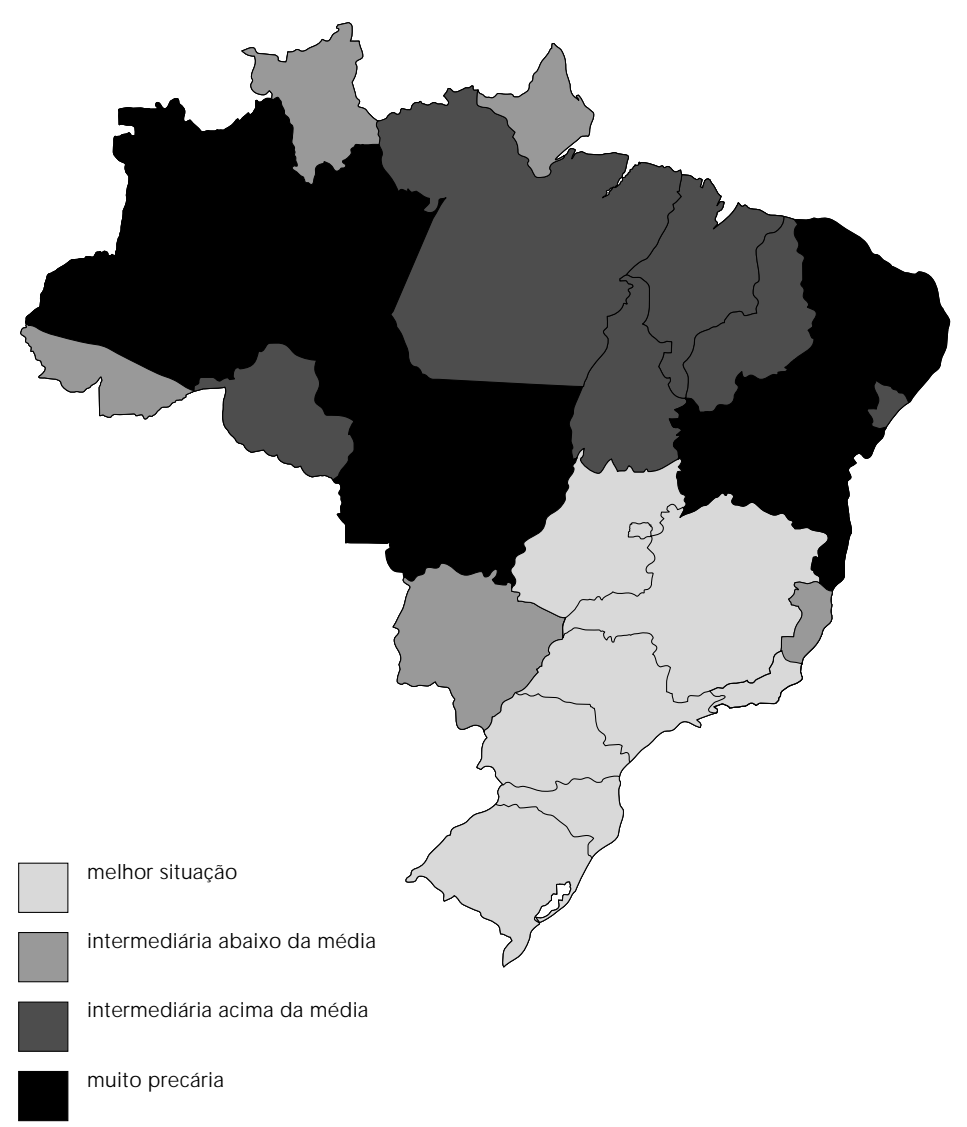

tivas finais muitas vezes não correspondem à UF como um todo, mas apenas às áreas do estado que possuem sistema regular de notificação. Questiona-se, por exemplo, a 16a posição apresentada pelo Piauí, relativamente ao índice sintetizador. Sabendo-se que, para 1991, o Piauí tem um coeficiente geral de mortalidade menor do que 3/ 1.000 habitantes (calculado por meio das informações de registro), é muito provável que o valor encontrado para o índice sintetizador não expresse, pelo menos de forma completa, a realidade deste estado. Da mesma forma, pode-se questionar a validade da classificação intermediária atribuída a Roraima e Amapá, diante da enorme subnotificação de óbitos apresentada por estas U Fs.

Além disto, é preciso destacar que a mortalidade proporcional por causa foi construída
Tabela 5

Escores do índice sintetizador e postos segundo Unidade da Federação, 1991.

\begin{tabular}{|c|c|c|}
\hline UF & Índice Sintetizador & Postos \\
\hline RO & 0,293 & 14 \\
\hline$A C$ & 0,040 & 13 \\
\hline$A M$ & 0,832 & 21 \\
\hline $\mathrm{RR}$ & $-0,339$ & 12 \\
\hline PA & 0,573 & 18 \\
\hline AP & $-0,445$ & 10 \\
\hline TO & 0,499 & 17 \\
\hline $\mathrm{MA}$ & 0,690 & 19 \\
\hline $\mathrm{PI}$ & 0,487 & 16 \\
\hline CE & 1,616 & 26 \\
\hline RN & 0,916 & 23 \\
\hline PB & 1,086 & 25 \\
\hline PE & 0,731 & 20 \\
\hline$A L$ & 1,829 & 27 \\
\hline SE & 0,399 & 15 \\
\hline BA & 0,839 & 22 \\
\hline MG & $-0,953$ & 6 \\
\hline ES & $-0,820$ & 9 \\
\hline RJ & $-1,350$ & 4 \\
\hline SP & $-1,464$ & 3 \\
\hline PR & $-0,828$ & 8 \\
\hline $\mathrm{SC}$ & $-0,984$ & 5 \\
\hline RS & $-1,537$ & 2 \\
\hline MS & $-0,442$ & 11 \\
\hline MT & 0,920 & 24 \\
\hline GO & $-0,916$ & 7 \\
\hline DF & $-1,673$ & 1 \\
\hline
\end{tabular}

indiretamente, através dos óbitos que têm definição da causa básica, o que pode ter causado distorções nos indicadores, justamente nos estados com condições mais precárias. Nas UFs onde há grande freqüência de mortes maldefinidas, a proporção de óbitos por diarréia entre os menores de um ano foi, provavelmente, subestimada, já que é esperado que, entre os óbitos que não têm definição da causa básica, haja uma proporção maior ainda de mortes atribuídas à diarréia.

Por conseguinte, as estimativas resultantes do índice sintetizador da mortalidade infantil devem ser interpretadas respeitando-se todas as limitações inerentes aos dados de registro, sendo, muitas vezes, mais adequado proceder a análise observando o comportamento das Unidades da Federação não só para o índice 
Tabela 6

Postos atribuídos aos indicadores de saúde segundo unidade da federação, 1991.

\begin{tabular}{|c|c|c|c|c|c|}
\hline UF & $\begin{array}{l}\text { Proporção (\%) } \\
\text { anomalias congênitas }\end{array}$ & $\begin{array}{l}\text { Proporção (\%) } \\
\text { diarréia }\end{array}$ & $\begin{array}{l}\text { Proporção (\%) } \\
\text { mal-definidas }\end{array}$ & $\begin{array}{l}\text { Cobertura } \\
\text { de registro (\%) }\end{array}$ & $\begin{array}{l}\text { Proporção (\%) } \\
\text { pós-neonatal }\end{array}$ \\
\hline DF & 1 & 4 & 1 & 1 & 3 \\
\hline RS & 3 & 1 & 4 & 1 & 6 \\
\hline SP & 6 & 2 & 3 & 1 & 1 \\
\hline RJ & 8 & 3 & 5 & 1 & 2 \\
\hline SC & 2 & 9 & 13 & 8 & 11 \\
\hline MG & 10 & 6 & 7 & 5 & 5 \\
\hline GO & 4 & 8 & 10 & 11 & 7 \\
\hline PR & 5 & 14 & 9 & 6 & 9 \\
\hline ES & 7 & 7 & 16 & 7 & 10 \\
\hline$A P$ & 14 & 12 & 2 & 8 & 4 \\
\hline MS & 12 & 10 & 6 & 10 & 12 \\
\hline $\mathrm{RR}$ & 13 & 5 & 8 & 22 & 8 \\
\hline$A C$ & 11 & 13 & 18 & 17 & 17 \\
\hline RO & 19 & 16 & 12 & 14 & 18 \\
\hline SE & 24 & 11 & 22 & 16 & 16 \\
\hline PI & 25 & 18 & 11 & 26 & 13 \\
\hline TO & 11 & 15 & 20 & 25 & 20 \\
\hline PA & 17 & 25 & 17 & 21 & 14 \\
\hline MA & 23 & 24 & 15 & 27 & 15 \\
\hline $\mathrm{PE}$ & 16 & 20 & 24 & 9 & 21 \\
\hline AM & 18 & 22 & 23 & 20 & 19 \\
\hline BA & 20 & 17 & 19 & 21 & 24 \\
\hline RN & 22 & 23 & 21 & 23 & 22 \\
\hline $\mathrm{MT}$ & 27 & 19 & 14 & 15 & 23 \\
\hline PB & 15 & 21 & 26 & 12 & 25 \\
\hline CE & 21 & 27 & 25 & 24 & 26 \\
\hline$A L$ & 26 & 26 & 27 & 13 & 27 \\
\hline
\end{tabular}

sintetizador ou para cada indicador individualmente, mas também em relação à sua coerência no conjunto de indicadores.

Evolução temporal dos indicadores de saúde no Brasil

A evolução de três dos cinco indicadores de saúde sob estudo, calculados tomando-se por base as informações do Ministério da Saúde (MS, 1995) durante o período de 1979 a 1993, está apresentada na Tabela 7 segundo Unidade da Federação. Para uma melhor interpretação do comportamento dos indicadores por UF, estimou-se a taxa anual de variação (expressa em percentual), calculada através do coeficiente angular da regressão exponencial ajus- tada às observações correspondentes aos anos de 1979, 1983, 1987 e 1991.

No que diz respeito à proporção de mortes tardias, destaca-se a Região Sudeste, particularmente os Estados de São Paulo e Rio de Janeiro, apresentando as maiores taxas de decréscimo. Seguem-se alguns estados do Norte, como Amapá, Pará e Roraima; todos os estados do Sul e Goiás e Mato Grosso do Sul, do Centro-Oeste. Caracterizando bem o processo de desenvolvimento nacional, em típica divisão em dois Brasis, poucos estados do Nordeste apresentaram declínio significativo, tendo-se, inclusive, estabelecido aumentos para Paraíba, Alagoas e Bahia.

Já para a proporção de mortes por doenças infecciosas intestinais, o decréscimo foi geral e abrangente a quase todas as UFs. Em compor- 
Tabela 7

Evolução da proporção de óbitos infantis tardios, da proporção de óbitos por diarréia e da proporção de óbitos mal-definidos, Brasil, 1979 a 1991,

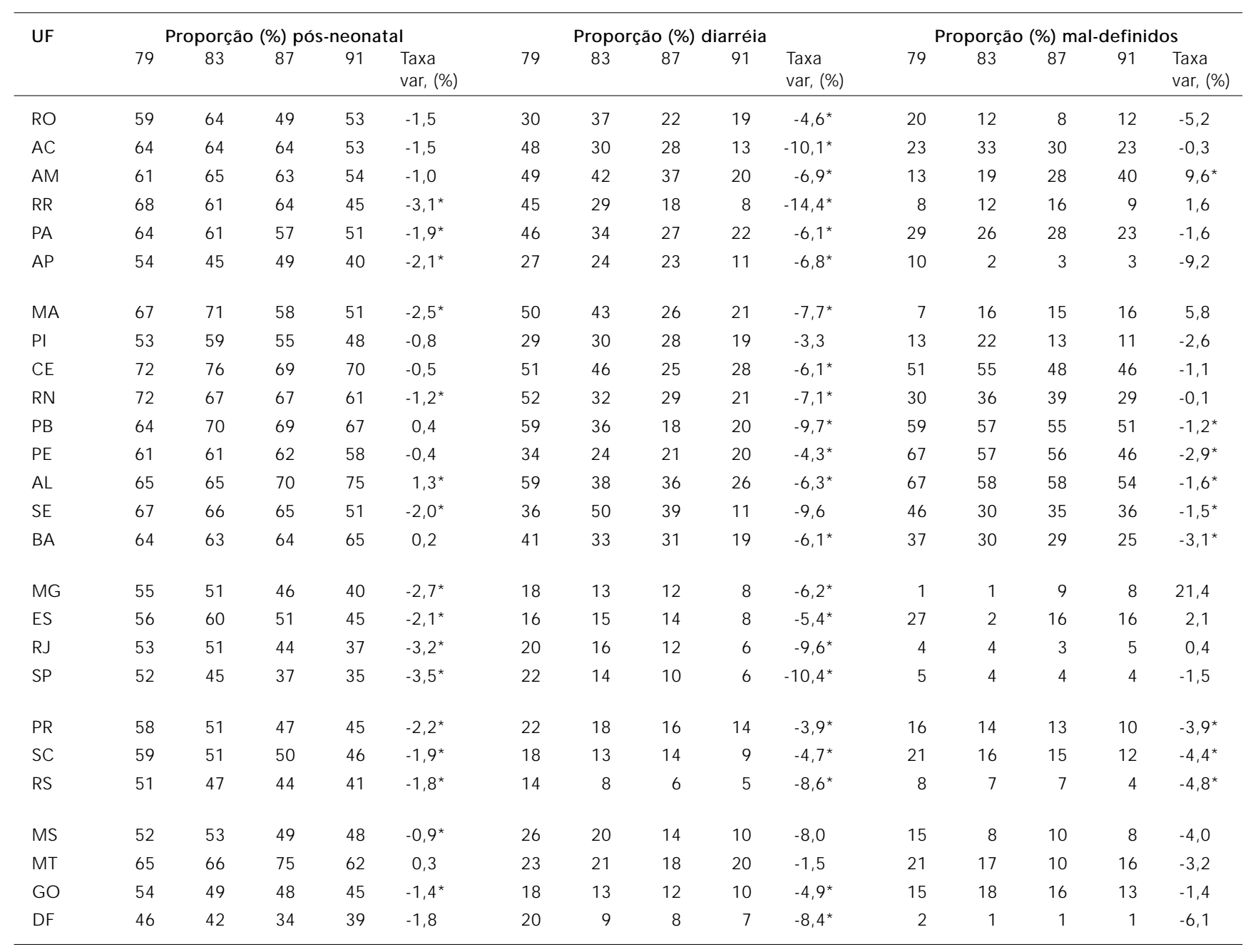

* Significativo ao nível de $5 \%$,

Fonte: Ministério da Saúde (1995).

tamento oposto, a proporção de óbitos classificados na categoria sinais e sintomas mal-definidos manteve-se estável na maioria dos estados, com exceções para a Região Sul e alguns estados do Nordeste, como Paraíba, Pernambuco, Alagoas e Bahia, que mostraram taxas significativas de declínio. Estes últimos, apesar do decréscimo, ainda têm proporções muito altas de óbitos sem definição da causa de morte, superiores, às vezes, a $50 \%$.

Os Estados do Rio Grande do Sul, São Paulo e Rio de Janeiro, por terem apresentado as meIhores situações em relação ao índice sintetizador da mortalidade infantil, mereceram atenção mais detida, com o intuito de confronto com a experiência de outros países (Tabela 8). Em todos os três estados, a evolução das taxas foi semelhante. Primeiramente, nota-se que o decréscimo na mortalidade infantil entre 1985 e 1993 foi inferior ao do começo da década (1979-1985), para ambos os componentes. Em segundo lugar, percebe-se que o declínio na mortalidade pós-neonatal foi bem maior que o da neonatal precoce.

Em análise comparativa, pode-se dizer que a queda da mortalidade tardia foi compatível com a de outros países, sem atingir, entretanto, os feitos de Portugal e Chile, que apresentaram decréscimos de $12 \%$ ao ano na década de 70 . Já em relação à mortalidade neonatal, nota-se que todos os países que conseguiram reduzir o nível de mortalidade infantil para menos do que 10/ 1.000 NV mostraram uma redução importante nos coeficientes na primeira semana de vida, cuja velocidade de queda passou a ser maior do que a do componente tardio. Diferen- 
temente destes países ou do Chile, que experimentou decréscimos importantes em ambos os componentes, os três estados brasileiros apresentam, ainda, redução pouco expressiva para a mortalidade nas primeiras horas.

Chama-se a atenção para o fato de que a velocidade de declínio da mortalidade pósneonatal diminuiu consideravelmente nos últimos anos nas três UFs sob análise. Este fato, aliado à pequena taxa de variação na mortalidade precoce, resultou em uma situação de redução limitada na mortalidade infantil no período de 1985 a 1993. Havendo continuidade deste comportamento, poder-se-á chegar, até mesmo, a uma estagnação no processo de queda do risco de morrer entre os menores de um ano.

\section{Comentários finais}

Segundo as estimativas do Banco Mundial, a mortalidade infantil no Brasil apresentou taxa de decréscimo de 2,2\% ao ano durante a década de 80. Comparada, porém, à obtida por outros países, a queda não foi muito expressiva. Ainda que categorizado entre os países de renda média, o Brasil, em comportamento semeIhante ao das populações de baixa renda, demonstrou declive inferior ao grupo em que é enquadrado e à maioria dos seus vizinhos na América do Sul, com raras exceções, como o Paraguai (The World Bank, 1995).

À luz da evolução da mortalidade infantil no País na última década, é interessante retomar a discussão de Bacha \& Klein (1986), que apontavam, no início dos anos 80 , para a existência de dois Brasis, um que se encaminhava rumo ao nível dos países desenvolvidos, como a Bélgica, e outro que permanecia em condições precárias de vida, tal como a Índia. É igualmente pertinente agregar a esta discussão a pesquisa recente do Ipea (1996), que, através da elaboração de um índice de desenvolvimento humano, veio a destacar um terceiro Brasil, caracterizado por ter um nível mediano de desenvolvimento.

De acordo com a análise dos dados de óbitos em menores de um ano realizada neste artigo, mostrou-se, sem dúvidas, que grande parte do Brasil, no início dos anos 90, continuava a apresentar condições muito deficientes. Compreendendo a maioria dos estados do Nordeste, algumas UFs da Região Norte e o Mato Grosso, esta parte teve descenso da mortalidade infantil em ritmo menor que o restante do País e permaneceu com um padrão similar ao de sociedades muito pobres. Demonstrou altas taxas de
Tabela 8

Taxas de variação da mortalidade neonatal precoce e pós-neonatal para países, estados do Brasil e anos selecionados.

\begin{tabular}{|c|c|c|c|c|}
\hline País & Ano & $\begin{array}{l}\text { CMI } \\
(/ 1,000)\end{array}$ & $\begin{array}{l}\text { Variação }(\%) \\
0-6 \text { dias }\end{array}$ & $\begin{array}{l}\text { Variação (\%) } \\
\text { pós-neonatal }\end{array}$ \\
\hline \multirow[t]{5}{*}{ J apão 1} & 1960 & 30,7 & - & - \\
\hline & 1970 & 13,1 & $-4,7$ & $-11,1$ \\
\hline & 1980 & 7,5 & $-5,3$ & $-5,5$ \\
\hline & 1986 & 5,2 & $-8,8$ & $-3,6$ \\
\hline & 1992 & 4,5 & $-4,1$ & 0,0 \\
\hline \multirow[t]{5}{*}{ Bélgical } & 1960 & 31,2 & - & - \\
\hline & 1970 & 21,1 & $-3,3$ & $-4,5$ \\
\hline & 1975 & 16,2 & $-4,0$ & $-9,0$ \\
\hline & 1979 & 12,3 & $-9,3$ & $-1,8$ \\
\hline & 1989 & 8,5 & $-8,1$ & $-0,5$ \\
\hline \multirow[t]{5}{*}{ Estados Unidos1 } & 1960 & 26,0 & - & - \\
\hline & 1969 & 21,0 & $-1,9$ & $-3,6$ \\
\hline & 1974 & 16,7 & $-5,5$ & $-3,7$ \\
\hline & 1984 & 10,8 & $-6,0$ & $-1,5$ \\
\hline & 1991 & 8,9 & $-2,9$ & $-2,0$ \\
\hline \multirow[t]{5}{*}{ Portugal1 } & 1960 & 77,5 & - & - \\
\hline & 1971 & 49,8 & $+0,5$ & $-5,4$ \\
\hline & 1979 & 26,0 & $-3,0$ & $-12,1$ \\
\hline & 1985 & 17,8 & $-3,3$ & $-9,9$ \\
\hline & 1993 & 8,7 & $-11,4$ & $-7,2$ \\
\hline \multirow[t]{5}{*}{ Polônia1 } & 1960 & 56,8 & - & - \\
\hline & 1970 & 33,2 & $-2,1$ & $-8,3$ \\
\hline & 1980 & 21,3 & $-3,1$ & $-5,3$ \\
\hline & 1985 & 22,1 & $+3,7$ & $-4,2$ \\
\hline & 1994 & 13,4 & $-6,1$ & $-6,0$ \\
\hline \multirow[t]{5}{*}{ Bulgária1 } & 1961 & 37,8 & - & - \\
\hline & 1970 & 27,3 & $-1,4$ & $-4,1$ \\
\hline & 1980 & 20,2 & $-1,9$ & $-3,5$ \\
\hline & 1985 & 15,2 & $-5,5$ & $-7,2$ \\
\hline & 1993 & 15,5 & $+0,8$ & $-0,2$ \\
\hline \multirow[t]{3}{*}{ Chile ${ }^{1}$} & 1974 & 63,3 & - & - \\
\hline & 1980 & 36,6 & $-3,4$ & $-12,0$ \\
\hline & 1989 & 17,1 & $-7,4$ & $-9,2$ \\
\hline \multirow[t]{3}{*}{ Rio Grande do Sul2 } & 1979 & 41,0 & - & - \\
\hline & 1985 & 26,8 & $-4,4$ & $-8,5$ \\
\hline & 1993 & 20,2 & $-2,8$ & $-4,3$ \\
\hline \multirow[t]{3}{*}{ São Paulo2 } & 1979 & 53,4 & - & - \\
\hline & 1985 & 35,0 & $-2,0$ & $-10,2$ \\
\hline & 1993 & 26,2 & $-1,7$ & $-5,8$ \\
\hline \multirow[t]{3}{*}{ Rio de J aneiro ${ }^{2}$} & 1979 & 50,8 & - & - \\
\hline & 1985 & 36,0 & $-1,6$ & $-8,3$ \\
\hline & 1993 & 29,0 & $-0,4$ & $-4,8$ \\
\hline
\end{tabular}

Fontes:1 - World Health Organization (1963; 1973; 1976; 1982; 1987; 1992; 1995); 2 - MS (1995). 
subnotificação, grandes proporções de óbitos sem definição da causa básica, elevados percentuais de mortes por doenças intestinais e não alcançou redução significativa na mortalidade no período pós-neonatal.

Se é certo que uma parte do País persiste com padrão semelhante ao da Índia, também não é dos mais otimistas o quadro apresentado pelo outro Brasil. Através do índice sintetizador da mortalidade infantil, verificou-se que apenas o Distrito Federal e o Rio Grande do Sul mostraram melhores condições do que São Paulo que, em 1991, apresentava CMI de 27/ 1.000 NV (Seade, 1993). Tendo-se em conta que estes estados brasileiros, em situação privilegiada em relação aos demais, apresentam coeficientes no mesmo patamar de países como o Chile, a Argentina, a Venezuela e o Uruguai, percebe-se que ainda é muito longo o caminho a ser percorrido para alcançar o mundo desenvolvido.

Sendo assim, a julgar pela mortalidade infantil, revela-se pouco convincente caracterizar um Brasil intermediário que se compararia à Bulgária, cujo CMI é de 15/ 1.000 NV e cuja mortalidade proporcional por diarréia é de apenas $2 \%$. Da mesma forma, diante dos valores tão contrastantes obtidos para todos os indicadores de saúde aqui trabalhados, não faz muito nexo agregar em um mesmo grupo os Estados do Amazonas e Minas Gerais, ou estabelecer que Roraima tem esperança de vida maior do que o Rio de Janeiro, conforme apontado pelo Relatório sobre o Desenvolvimento Humano no Brasil (IPEA, 1996).

Contudo, em termos relativos, é bem verdade que um grupo de estados da Região Norte já conseguiu sair do marasmo nordestino e apresenta, pelo menos, coeficientes de mortalidade infantil em nível inferior a 50/ 1.000 NV. Estes estados vêm tomando a posição central ocupada anteriormente pela Região Centro-Oeste, que, de acordo com os indicadores analisados, partiu-se em duas regiões que tomaram direções opostas: enquanto uma, formada pelo Distrito Federal, Goiás e M ato Grosso do Sul, classifica-se, atualmente, entre os melhores postos, a outra, composta por M ato Grosso e Tocantins (ex-Goiás), acompanhou o lado menos desenvolvido da nação.

Da análise realizada para os estados que tiveram melhor desempenho (Rio Grande do Sul, São Paulo e Rio de Janeiro), conclui-se que o CMI de 8/ 1.000 NV apresentado pela Bélgica ainda é um ideal longínquo. Em todos os países que conseguiram reduzir expressivamente a mortalidade infantil, verificou-se uma grande diminuição do componente precoce, parti- cularmente nos primeiros dias de vida, período em que a criança ainda está sob cuidados médicos (Kalter, 1991; Nakamura et al., 1991). Em contraposição, em nenhum dos três estados brasileiros, houve indícios de progresso em relação à mortalidade infantil precoce. Como demonstrado por Carvalho (1993), procedimentos simples e de baixo custo, ligados à atenção médico-hospitalar no momento do parto, seriam capazes de determinar reduções substanciais na mortalidade infantil.

De maneira geral, o confronto com a experiência internacional leva, inexoravelmente, a constatar que o declive da mortalidade infantil no País foi discreto, e que nem o Nordeste se desvencilhou da imagem da Índia, nem a parte rica chegou perto da Bélgica. Fica evidente também que o rumo a ser seguido envolve intervenções específicas sobre ambos os componentes, devendo-se não só dar continuidade às políticas públicas que, ao menos parcialmente, mostraram-se efetivas e alcançaram bons resultados, mas resgatar de imediato os programas de pré-natal e de atenção ao parto e ao recém-nascido.

Como palavras finais, tem-se a dizer que é triste mostrar que o desenvolvimento social do Brasil ficou muito aquém do de outros países durante a década de 80 e deveras constrangedor apresentar a realidade crua das nossas estatísticas de saúde, flagrando as precárias condições de vida de grande parte de nossa população. Porém, muito mais desolador, é colorir um quadro patético para uma sociedade desinformada que, percorrendo um caminho singular de desigualdades, não sabe, até hoje, reivindicar o direito à vida de suas crianças. 


\section{Agradecimentos}

Agradecemos as valiosas contribuições dadas pelos revisores do artigo, sobretudo no que diz respeito às informações referentes ao fluxo das declarações de óbito.

\section{Referências}

BACHA, E. \& KLEIN, H., 1986. A Transi ção Incomple ta: Brasil desde 1945. Rio de Janeiro: Paz eTerra.

BANCO MUNDIAL, 1993. Relatório sobre o Desenvolvimento Mundial. Rio de Janeiro: Fundação Getúlio Vargas.

BANCO MUNDIAL, 1994. Relatório sobre o Desenvolvimento Mundial. Rio de Janeiro: Fundação Getúlio Vargas.

BANCO MUNDIAL, 1995. Relatório sobre o Desenvolvimento Mundial. Rio de Janeiro: Fundação Getúlio Vargas.

BARROS, F. C.; VICTORA, C. G.; TEIXEIRA, A. M. B. \& PUERTO-FILHO, M., 1985. Mortalidade perinatal e infantil em Pelotas, Rio Grande do Sul: nossas estatísticas são confiáveis? Caderno de Saúde Pública, 1:348-358.

BECKER, R. A. \& LECHTIG, A., 1986. Brasil: Evolução da Mortalidade Infantil no Período 1977-1984. Brasília: Centro de Documentação, Ministério da Saúde. Série C: Estudos e Projetos, 3.

CARVALHO, M. L., 1993. Mortalidade Neonatal eAspectos da Qualidade da Atenção à Saúde na Região Metropolitana do Rio de Janeiro 19861987. Dissertação de Mestrado, Rio de Janeiro: Escola Nacional de Saúde Pública, Fundação Oswaldo Cruz.

CCDP (Centro da OMS para Classificação de Doenças em Português), 1978. Classificação Estatística Internacional de Doenças, Lesões e Causas de óbito: 9a revisão (1975). São Paulo: CCDP.

FIBGE (Fundação Instituto Brasileiro de Geografia e Estatística), 1981. Estatísticas do Registro Civil. Vol. 6, Rio de Janeiro: FIBGE.

FIBGE (Fundação Instituto Brasileiro de Geografia e Estatística), 1982. Estatísticas do Registro Civil. Vol. 7, Rio de Janeiro: FIBGE.

FIBGE (Fundação Instituto Brasileiro de Geografia e Estatística), 1983. Estatísticas do Registro Civil. Vol. 8, Rio de Janeiro: FIBGE.

FIBGE (Fundação Instituto Brasileiro de Geografia e Estatística), 1984. Estatísticas do Registro Civil. Vol. 9, Rio de Janeiro: FIBGE.

FIBGE (Fundação Instituto Brasileiro de Geografia e Estatística), 1985. Estatísticas do Registro Civil. Vol. 10, Rio de Janeiro: FIBGE.

FIBGE (Fundação Instituto Brasileiro de Geografia e Estatística), 1986. Estatísticas do Registro Civil. Vol. 11, Rio de Janeiro: FIBGE.

FIBGE (Fundação Instituto Brasileiro de Geografia e Estatística), 1987. Estatísticas do Registro Civil. Vol. 12, Rio de Janeiro: FIBGE.
FIBGE (Fundação Instituto Brasileiro de Geografia e Estatística), 1988. Estatísticas do Registro Civil. Vol. 13, Rio de Janeiro: FIBGE.

FIBGE (Fundação Instituto Brasileiro de Geografia e Estatística), 1989. Estatísticas do Registro Civil. Vol. 14, Rio de Janeiro: FIBGE.

FIBGE (Fundação Instituto Brasileiro de Geografia e Estatística), 1990. Estatísticas do Registro Civil. Vol. 15, Rio de Janeiro: FIBGE.

FIBGE (Fundação Instituto Brasileiro de Geografia e Estatística), 1991. Estatísticas do Registro Civil. Vol. 16, Rio de Janeiro: FIBGE.

FIBGE (Fundação Instituto Brasileiro de Geografia e Estatística), 1992. Estatísticas do Registro Civil. Vol. 17, Rio de Janeiro: FIBGE.

FIBGE (Fundação Instituto Brasileiro de Geografia e Estatística), 1993. Estatísticas do Registro Civil. Vol. 18, Rio de Janeiro: FIBGE.

FIBGE (Fundação Instituto Brasileiro de Geografia e Estatística), 1994. Estatísticas do Registro Civil. Vol. 19, Rio de Janeiro: FIBGE.

FIBGE (Fundação Instituto Brasileiro de Geografia e Estatística), 1995. Estatísticas do Registro Civil. Vol. 20, Rio de Janeiro: FIBGE.

GREEN, P. E., 1978. Analyzing Multivariate Data. Hinsdale: The Dryden Press.

HANOLUWKA, H. E., 1987. Measuring the health status of a population: current state of the art. Population Bulletin of the United Nations, 23/24:56-75.

IPEA (Instituto de Pesquisa Econômica Aplicada), 1996. Relatório sobre o Desenvolvimento Humano no Brasil. Brasília: Programa das Nações Unidas para o Desenvolvimento.

KALTER, H., 1991. Five decade international trends in the relation of perinatal mortality and congenital malformations: stillbirth and neonatal death compared. International Journal of Epidemiology, 20:173-179.

LAU RENTI, R.; MELLO JORGE, M. H. P.; LEBRÃO, M. L. \& GOTLIEB, S. L. D., 1985. Estatísticas deSaúde. São Paulo: Editora Pedagógica e Universitária.

LEE, K.; PANETH, N.; GARTNER, L. M.; PEARLMAN, M. A. \& GRUSS, L., 1980. Neonatal mortality: an analysis of the recent improvement in the United States. American Journal of Public Health, 70:1521.

MELLO JORGE, M. H. P., 1983. Sub-registro dos eventos vitais. Revista de Saúde Pública, 17:148-151.

MELLO JORGE, M. H. P., 1987. Sub-registro de óbitos e aspectos da legislação sobre mortalidade. II Reunião Nacional do Subsistema de Informação sobre 
Mortalidade. Brasília. (mimeo.)

MS (Ministério da Saúde), 1982. Estatísticas de Mortalidade Brasil 1979. Brasília: Centro de Documentação.

MS (Ministério da Saúde), 1995. Sistema de Informações sobre Mortalidade. Brasília: MS.

NAKAMURA, Y.; NAGAI, M. \&YANAGAWA, H.; 1991. A characteristic change in infant mortality rate decrease in Japan. Public Health, 105:145-151.

POLLARD, A. H.; YUSUF, F. \& POLLARD, G. N., 1974. Demographic Techniques. Sidney: Pergamon Press.

SEADE (Sistema Estadual de Análise de Dados), 1993. Anuário Estatístico do Estado de São Paulo 1992. São Paulo: SEADE.

SIMÕES, C. C. S. \& OLIVEIRA, L. A. P., 1986. Evolução da mortalidade infantil. In: Perfil Estatístico de Crianças e Mães no Brasil - Aspectos SócioEconômicos da Mortalidade Infantil em Áreas Urbanas (FIBGE, org.), pp. 29-48. Rio de Janeiro: FIBGE.
SZWARCWALD, C. L. \& CASTILHO, E. A., 1995. Estimativas da mortalidade infantil no Brasil, década de 80: proposta de procedimento metodológico. Revista de Saúde Pública, 29:451-462.

THE WORLD BANK, 1995. Social Indicators of Development 1995. Baltimore: The John Hopkins University Press.

WHO (World Health Organization), 1963. World Health Statistics Annual. Geneve: WHO.

WHO (World Health Organization), 1973. World Health Statistics Annual. Geneve: WHO.

WHO (World Health Organization), 1976. World Health Statistics Annual. Geneve: WHO.

WHO (World Health Organization), 1982. World Health Statistics Annual. Geneve: WHO.

WHO (World Health Organization), 1987. World Health Statistics Annual. Geneve: WHO.

WHO (World Health Organization), 1992. World Health Statistics Annual. Geneve: WHO.

WHO (World Health Organization), 1995. World Health Statistics Annual. Geneve: WHO. 